\title{
Identification of Coronary Heart Disease through Iris using Gray Level Co-occurrence Matrix and Support Vector Machine Classification
}

\author{
Vincentius Abdi Gunawan ${ }^{1}$ * \\ Department of Informatics Engineering \\ Universitas Palangka Raya \\ Palangka Raya, Indonesia
}

\author{
Leonardus Sandy Ade Putra ${ }^{2}$, Fitri Imansyah ${ }^{3}$, Eka \\ Kusumawardhani ${ }^{4}$ \\ Department of Electrical Engineering \\ Universitas Tanjungpura \\ Pontianak, Indonesia
}

\begin{abstract}
Now-a-days, coronary heart disease is one of the deadliest diseases in the world. An unfavorable lifestyle, lack of physical activity, and consuming tobacco are the causes of coronary heart disease aside from genetic inheritance. Sometimes the patient does not know whether he has abnormalities in heart function or not. Therefore, this study proposes a system that can detect heart abnormalities through the iris, known as the Iridology method. The system is designed automatically in the iris detection to the classification results. Feature extraction using five characteristics is applied to the Gray Level Cooccurrence Matrix (GLCM) method. The classification process uses the Support Vector Machine (SVM) with linear kernel variation, Polynomial, and Gaussian to obtain the best accuracy in the system. From the system simulation results, the use of the Gaussian kernel can be relied on in the classification of iris conditions with an accuracy rate of $91 \%$, then the Polynomial kernel accuracy reaches $\mathbf{8 9 \%}$, and the linear kernel accuracy reaches $87 \%$. This study has succeeded in detecting heart conditions through the iris by dividing the iris into normal iris and abnormal iris.
\end{abstract}

Keywords-Iris; iridology; coronary heart; circle hough transform; gray level co-occurrence matrix; support vector machine

\section{INTRODUCTION}

Coronary heart disease is the number one cause of death worldwide. According to data from the World Health Organization (WHO), there are 17 million people in the world who die from coronary heart disease. In Indonesia, coronary heart disease is the highest cause of death after stroke, with a mortality rate of $12.9 \%$ in 2014 [1]. Every year there are 1.9 million people die of coronary heart disease due to consuming tobacco [2]. An unhealthy lifestyle and lack of physical activity are the leading causes of coronary heart disease [3]. The death rate is higher among the older age population [4]. Consuming foods high in carbohydrates or fat and obesity are factors that cause constriction of blood vessels in the heart.

Examination to determine coronary heart disease will be advised by checking with an Electrocardiogram (EKG), which uses electricity to determine heart rhythm. The use of echocardiography is also sometimes done to see the part, pump function, and valve function of the heart. Taking some actions to check the current heart condition costs quite a bit so that a person will be reluctant to examine his heart condition.

Early prevention can be done to reduce the risk of coronary heart disease, namely by consuming enough fruits and vegetables every day [5], exercising or doing physical activities regularly in daily life, and do not consume tobacco [1].

Nowadays, technology to detect heart conditions from an early stage has been carried out by scientists in the medical field and computing technology. One of them is knowing the heart condition through the eye's iris, which is directly connected to the brain [6]. The brain is a human organ that receives $15 \%$ of blood flow from the heart and accounts for $20 \%$ of oxygen consumption in the body, making it susceptible to vascularization in the human brain [7].

The iris is one of the unique organs in the human body. Iris is usually used as electronic security or biometric identification system $[8,9]$. Nowadays, the research on the iris in the medical field is increasingly widespread and is being seriously studied by experts. Iris can provide information about the condition of human organs, known as the Iridology method.

Iridology is based on the analysis of one of the most complex tissue structures contained in the iris. This method can determine the condition of organs and systems in the body from the marks on the iris. Iridology cannot diagnose a disease but instead helps to identify existing or any potential problems in a particular organ [10]. According to Iridologists, the condition of the overall organ is reflected on the surface of the iris [11]. In healthcare practitioners, the iris is used to determine the systemic health, innate strengths, or weaknesses of an individual's personality [12]. Image processing and data mining processing techniques have been used as disease diagnosis tools in biomedical applications. There are parameters in improving the quality of the iris diagnosis technique, especially by using iris images by focusing on the PRISMA-ScR guidelines [13]. Based on this statement, the research obtained $89.63 \%$ classification accuracy of diabetic patients with iris diagnosis [14]. Heydari M. and Teimouri M. have also conducted a test on type 2 diabetic patients and obtained the highest accuracy rate of $97.44 \%$ using Artificial

*Corresponding Author 
Neural Networks in Iran. Young-WoolLim and YoungBaelPark [11] have also conducted a study to examine the relationship between the iris and the characteristics differences of each individual. The results show that iris parameters can be used more definitely related to characteristics than functional changes. Research conducted by M. Gopalan and Gopal S. Pollai stated that iridology could be used to determine the health of humans organs contained in the character of the iris [12], Recognition of the iris identifies biometrics and can diagnose the presence of cholesterol in the blood [15]. Iris is used in providing information of human's physical condition to determine vehicle driver fatigue by detecting the behavior of the iris that has been carried out by [16], with an accuracy of $80 \%$. Research by [17] has diagnosed the heart with the iris using Principal Component Analysis (PCA) and has achieved 80\% success using the SVM classification. The use of the GLCM method was also carried out by [18] by using four characteristics of feature extraction with an accuracy rate of $85.6 \%$.

Previous studies have used the iris as an early diagnosis in detecting abnormal conditions in human organs. The use of different methods leads to different results in identification accuracy. The GLCM method using six characteristics of feature extraction has not been carried out until now. So this is a new scope for in-depth research to be carried out in the identification process. Separating the iris from the rest of the eye is carried out automatically using CHT by utilizing circle edge detection, an important part of the identification system designed in this paper.

In this study, iris samples were collected from healthy individuals and patients with coronary heart disease to explore new directions and methodologies in diagnosing coronary heart disease with computer imaging and machine learning techniques. The determination of the iris section has been carried out automatically using the Hough transformation method, which is considered more efficient in reading multiple iris samples [19]. Gray Level Co-occurrence Matrix as a texture extraction method was chosen in this study because of its ability to perform feature extraction in grayscale images properly [20]. The selection of GLCM as a feature extraction method is based on previous research that has not tested the condition of the heart through the iris using the six characteristics of GLCM. The GLCM method is considered to be better at reading images with grayscale values to obtain accurate results. Determination of the iris that reflects normal and abnormal heart conditions is carried out using the Support Vector Machine as a classification method. The SVM method can separate data into classes with linear solutions in high dimensions [21]. So that the final results of this study can help provide information on normal heart conditions and heart conditions with abnormal conditions through the iridology method. The system design flow can be shown in Fig. 1.

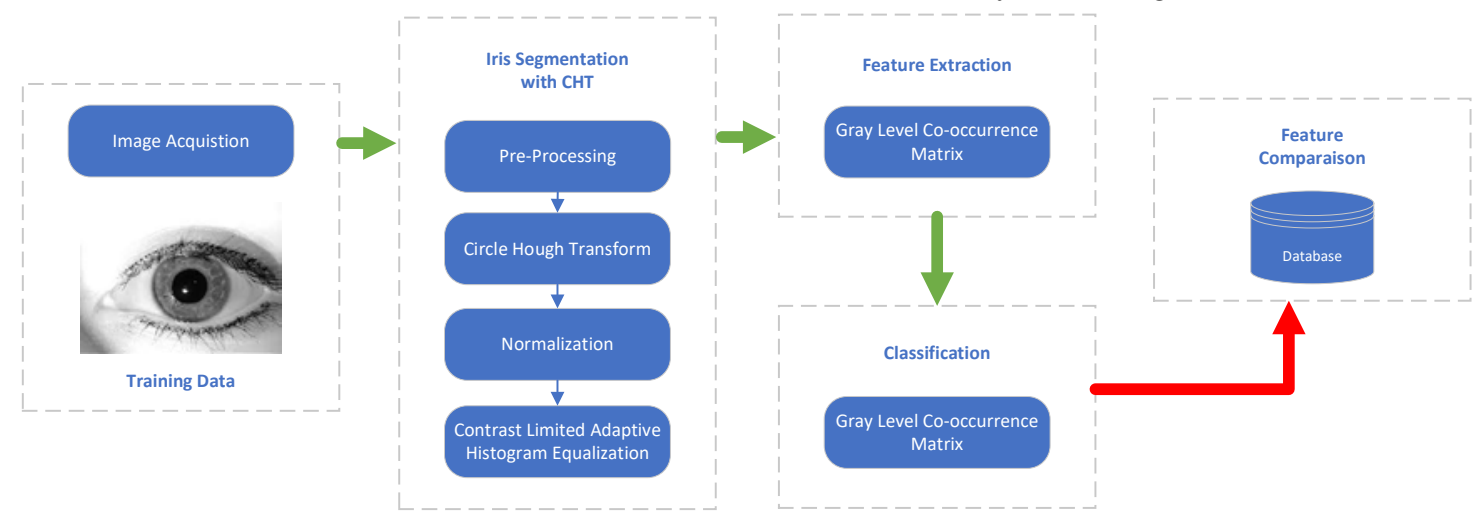

(a)
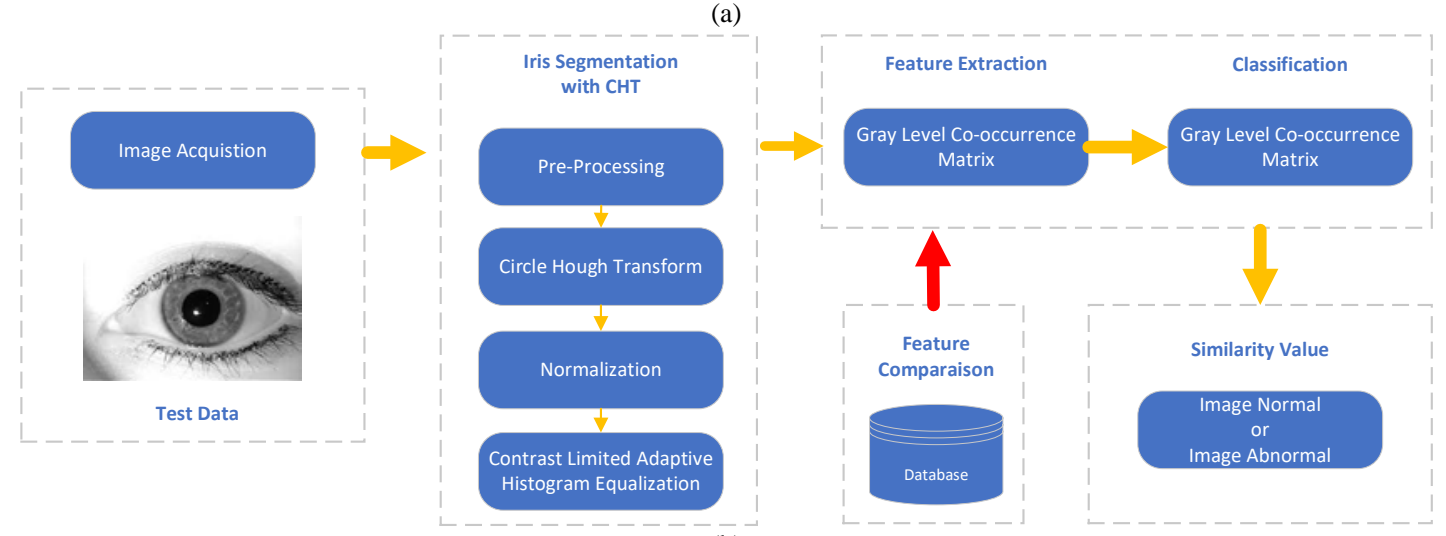

(b)

Fig. 1. Shows the Flow of the Designed System; a) the flow of Training on the Recognition System; b) Test Flow on the Recognition System. 


\section{RESEARCH METHOD}

\section{A. Iridology}

Iridology is known as a diagnostic method using the human iris. In the medical world, the iris can interpret the condition of the human organs [22]. Iridology divides the iris into 60 parts, and each part represents the condition or function of its organs. The right iris will reflect the condition of the right organs of the body, and vice versa, where the left iris will reflect the condition of the left organs [23].

Dr. Bernard Jensen has created a chart that describes each part of the 60 sectors into an image that is mirrored in a circular image like a clock and is divided into sectors according to the part of the iris that reflects the organ. The chart has been described according to the division in the left eye and right eye [10], [24].

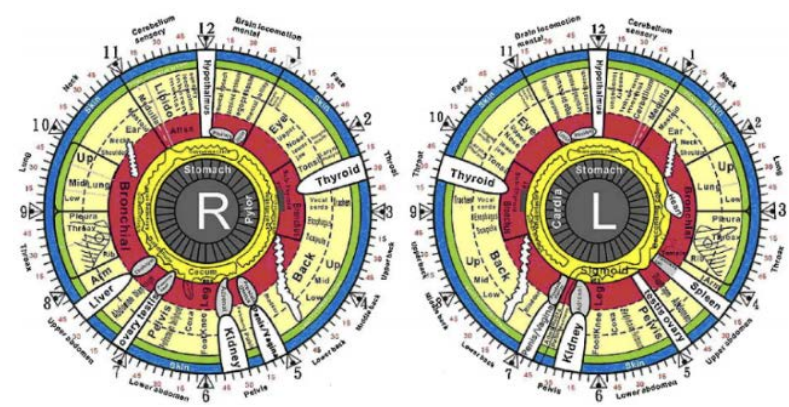

Fig. 2. Left Iris and Right Iris Iridology Chart [10].

We can see in Fig. 2, the position of the heart is on the left side of the iris. The location of the heart is reflected through the left iris, which is shown in the iris zone 02.00-03.15.

\section{B. Pre-Processing}

The image captured through a digital camera has an RGB (Red, Green, Blue) format, so it is necessary to do a preprocessing process. In the preprocessing the iris image will be converted from an RGB image to a Greyscale image. Grayscale images have a simpler color value with a color intensity of $0-255$ pixel thus shortening the computation time. The next step is to cut the unused part outside the iris, leaving a part that is close to the iris. Then, The eye image will be resized so that the entire image has the same pixel size when it enters the extraction process [25].

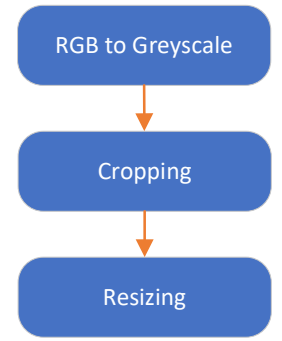

Fig. 3. Image Pre-Processing Process.

Fig. 3 shows the pre-processing chart. The resized image will be processed into the CHT method to obtain the iris and eliminate the pupil area.

\section{Circle Hough Transform}

Images that have gone through the RGB to Grayscale conversion process will be separated between the iris and other objects that are not used, especially the pupil. The CHT method is used to determine the iris part automatically without human assistance in determining the coordinates of the iris. $\mathrm{CHT}$ can detect circles in the iris image and know between the outer iris and the outer pupil.

$(x-a)^{2}+(y-b)^{2}=r^{2}$

Equation 1 describes the center circle $(a, b)$ and has a radius of $r$. With $(x, y)$ is a pixel at the edge of the circle, en it can be represented in the form of a circle as [26]:

$\left\{\begin{array}{l}x=a+r \cos \theta \\ y=b+r \sin \theta\end{array}\right.$

Fig. 4 describes the CHT method that is implemented into the iris image. The iris image at point (a) has RGB format, which needs to be converted into a greyscale format. The histogram in the grayscale image shows high values in several white areas of objects that occur from light reflections and black colors that have values from the middle pupil of the iris.

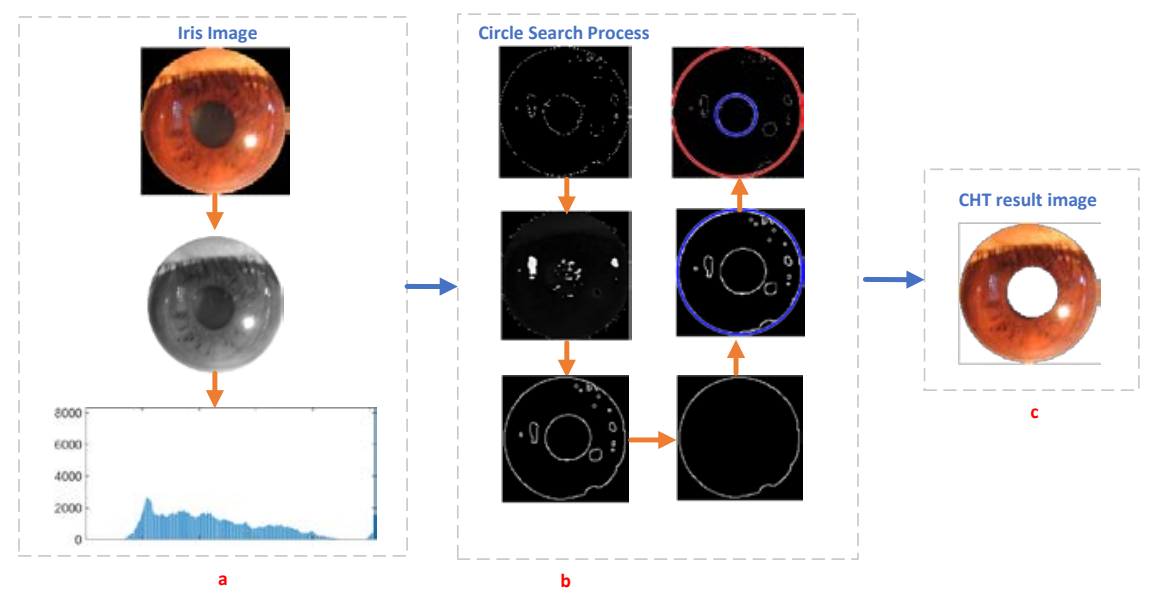

Fig. 4. CHT Process in Detecting Circles on the Iris; a) Iris Image in RGB Format is Converted into Grayscale Format along with Histogram Values; b) Circle Detection Process with CHT; c) the Results of the CHT Process. 
Then the image at point $b$ is an image display in determining the point of the circumference of the pupil and iris ring. If a part of the noise is not circular in this process, it will be removed. The image is then given a thickening process on the line to determine the circle in the image. Furthermore, the part that has been detected as a circle will be combined with the initial RGB image and generate an image like point c.

The CHT algorithm is used to separate the iris from the pupil and sclera. This automatic determination is carried out using edge detection in the form of a circle with a diameter value to determine the inner circle and outer circle of the iris. Edge detection is carried out to find objects with a diameter of less than $3 \mathrm{~mm}$ for the object removal process. Then the system will detect a circle with a diameter of more than $3 \mathrm{~mm}$ with a shape close to a perfect circle that is selected as the inner iris circle. In determining the outline of the outer iris circle, the system looks for the diameter of the circle measuring $12 \mathrm{~mm}$. Determination of the diameter of the circle is adjusted to the process of taking pictures using constant light so that the images in each data taken to have the same size.

\section{Normalization}

The texture in the image has coordinates that represent the dimensions of the iris image, such as pupil dilation [27].The iris image segmentation method aims to normalize the image in a different form but still has the exact resolution [28]. The iris can be modeled by two non-concentric circles and different textures within an iris circle. The center of the pupil can be used as a reference point for the circle on the iris [8]. A radial line runs through the area in the iris, known as radial resolution. Since the pupil is elastic to the iris, it is necessary to rescaling the reference point. The scaling equation is calculated based on the angle around the iris circle, with the equation:

$r^{\prime}=\sqrt{a \beta^{2}-a-r_{l}^{2}}+\sqrt{a \beta}$

where,

$a=o_{x}^{2}+o_{y}^{2}$

$\beta=\cos \left(\pi-\arctan \left(\frac{o_{y}}{o_{x}}\right)-\theta\right)$

$r^{\prime}$ is the distance between the pupil and the iris, while $\theta$ is the edge angle based on the radius of the iris. $o_{x}$ and $o_{y}$ is the displacement from the center of the pupil to the displacement of the center of the iris [29].

The Iris image that has a circular shape needs to be normalized based on the angle. The circular iris will be formed into a 2D array with horizontal dimensions at angles and vertical dimensions at radial. The circular iris will be formed into a 2D array with horizontal dimensions at angles and vertical dimensions at radial so that it will produce a rectangular or polar image shape as shown in Fig. 5 [30, 31].

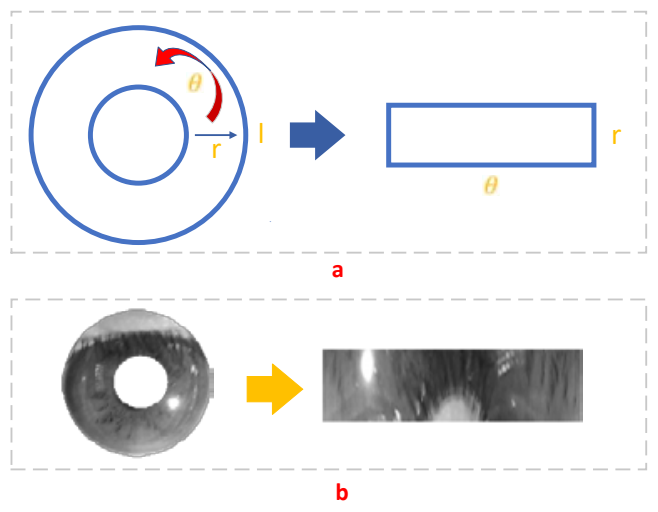

Fig. 5. Area of Circle to Rectangle Transformation of Daugman Model; a) Daugman Model; b) The Implementation Result of Iris Image.

\section{E. Contrast Limited Adaptive Histogram Equalization (CLAHE)}

CLAHE is a method to improve image quality by limiting the histogram value [32]. In this study, the CLAHE method is used to increase image intensity so that there will be a lot of detail that can be improved on the image. The results of using CLAHE are shown in Fig. 6.

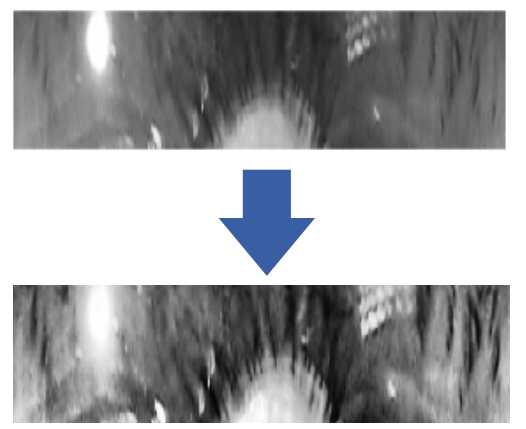

Fig. 6. Results of using the CLAHE Method on Polar Iris Images.

\section{F. Region of InterestA}

The process of diagnosing the heart through the iris requires certain parts so that it does not require the whole to be processed. According to Iridology, the iris that reflects the heart is on the left iris, as shown in the sector $02.00-03.15$ according to Fig. 1.

Region of Interest (ROI) is a process where we only need a specific part of the image to be processed. These sections will be cropped, leaving only the heart's reflection in the iris of the left, as shown in Fig. 7.

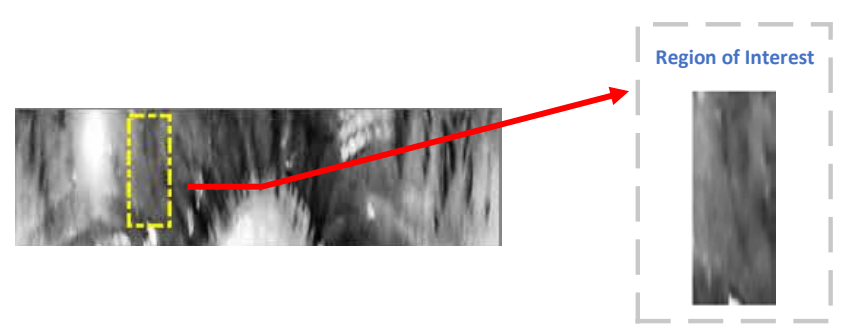

Fig. 7. Application of ROI of the Heart on the Iris Image. 


\section{G. Gray Level Co-occurrence Matrix (GLCM)}

GLCM is a texture analysis technique on images with a gray level. GLCM has a relationship between 2 neighboring pixels, which is determined by the intensity of gray, a certain distance, and angle. The equation for GLCM is shown below [33]:

$G_{(\Delta x, \Delta y)}(a, b)=\sum_{i=1}^{P} \sum_{j=1}^{Q} 1\{I(i, j)=a\}$ and $1\{I(+\Delta x, j+$ $\Delta y)=b\}$

$I(i, j)$ is the gray value of the column (i) and row (j) pixels, $(a, b)$ is a gray value that occurs at the same time as the calculation of $G_{(\Delta x, \Delta y)}(a, b)$. Then, $1\{I(+\Delta x, j+\Delta y)=b\}$ is the indicator of $\Delta x$ as a direction from $x$ and $\Delta y$ as a direction of $y$ which is determined by the distance of the $x$ and $y$. $P$ and $Q$ shows the rows and columns of the corresponding image. There are 4 angles used in GLCM, $0^{\circ}, 45^{\circ}, 90^{\circ}$ and $135^{\circ}$, described in Fig. 8.

The illustration in Fig. 9 is the use of GLCM for image pixels. Point a shows the use of distances that have a value of $d=1$ with 4 different directions where $d$ is the distance between pixels. Point $b$ shows the GLCM Usage calculation on an image with $\theta=0^{\circ}$ and the distance between pixels is 1 .
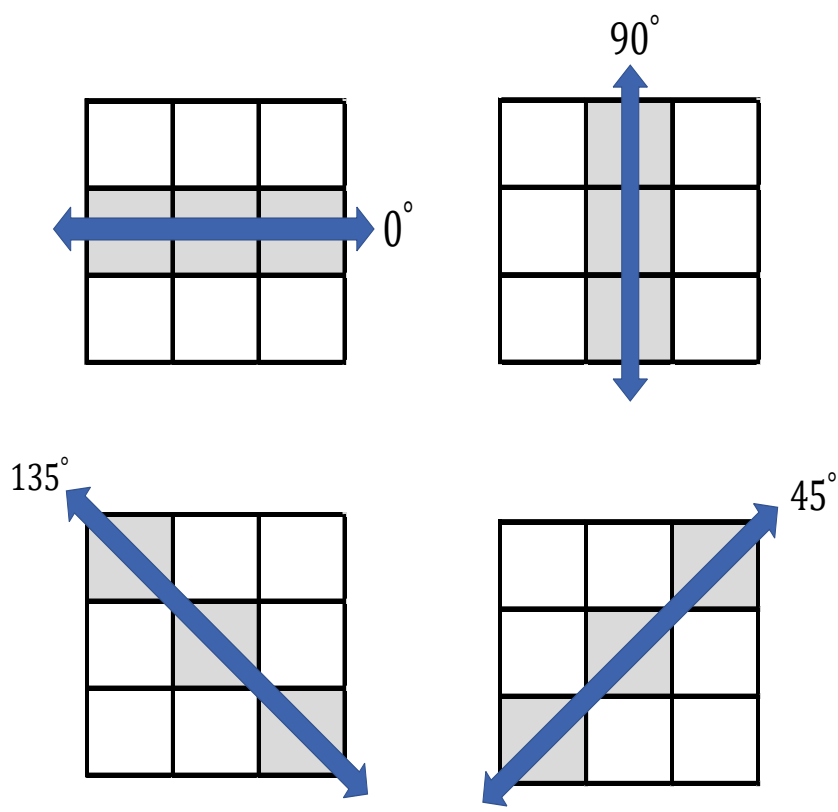

Fig. 8. Illustration of 4-Way Angle GLCM.

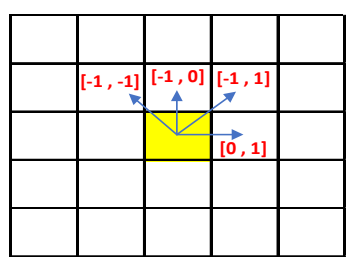

(a)

\begin{tabular}{|l|l|l|l|l|}
\hline 2 & 1 & 2 & 1 & 3 \\
\hline 3 & 2 & 3 & 4 & 4 \\
\hline 1 & 4 & 2 & 1 & 1 \\
\hline 3 & 1 & 2 & 1 & 2 \\
\hline 3 & 1 & 3 & 1 & 2 \\
\hline
\end{tabular}$\longrightarrow$\begin{tabular}{|c|c|c|c|c|}
\hline Pixel & 1 & 2 & 3 & 4 \\
\hline 1 & 1 & 3 & 1 & 1 \\
\hline 2 & 4 & 0 & 1 & 0 \\
\hline 3 & 3 & 1 & 0 & 1 \\
\hline 4 & 0 & 1 & 0 & 1 \\
\hline
\end{tabular}

(b)

Fig. 9. GLCM Angle Illustration; a) is an Illustration of the use of $\theta=$ $0^{\circ}, 45^{\circ}, 90^{\circ}, 135^{\circ}$ dan $d=1$; b) GLCM Usage Calculations for Images with $\theta=0^{\circ}$ and $d=1$.

The image that has been calculated for certain distances and angles will then be transposed to the values obtained, and then the GLCM matrix values are added to the transpose results. The results will be normalized using the following equation:

$G L C M_{\text {Norm }}=\frac{\text { GLCM }_{\text {Value }}}{\sum_{i}^{N} \text { GLCM }}$

where,

$G L C M_{\text {Value }}=$ value of each pixel

The normalization results can be used to determine the texture characteristics of the image by obtaining information, such as Contrast, Dissimilarity, Homogeneity, Angular Second Moment (ASM), Energy and Correlation. The function of the contrast characteristic is the calculation of the difference in intensity between adjacent pixels in the entire image. Dissimilarity is the process of measuring the difference in a texture with a significant value if it is random, and vice versa will have a small value if it is uniform. Homogeneity functions to show the homogeneity of intensity variations in the image. ASM is a uniformity measurement process that produces a high value if the pixel values are similar to each other and vice versa will have a low value if the pixel values are different. Energy is used to measure the concentration of intensity pairs in the matrix, and Correlation is a linearity measurement of several pixel pairs. The equation to obtain each characteristic is shown in Table I [34]: 
TABLE I. CHARACTERISTIC EQUATIONS ON GLCM

\begin{tabular}{|c|c|}
\hline $\begin{array}{l}\text { Texture Characteristics of } \\
\text { GLCM }\end{array}$ & Equation \\
\hline Contrast & $\sum_{a, b=0}^{\text {level-1 }} P_{a, b}(a-b)^{2}$ \\
\hline Dissimilarity & $\sum_{a, b=0}^{\text {level-1 }} P_{a, b}|a-b|$ \\
\hline Homogeneity & $\sum_{a, b=0}^{\text {level-1 }} \frac{P_{a, b}}{1+(a-b)^{2}}$ \\
\hline ASM & $\sum_{a, b=0}^{\text {level-1 }} P_{a, b}^{2}$ \\
\hline Energy & $\sum_{a, b=0}^{\text {level-1 }} P_{a, b}^{2}$ \\
\hline Correlation & $\begin{array}{l}\sum_{a, b=0}^{\text {level }-1} P_{a, b}\left[\frac{\left(a-\mu_{a}\right)\left(b-\mu_{b}\right)}{\sqrt{\left(\sigma_{a}^{2}\right)\left(\sigma_{b}^{2}\right)}}\right] \\
\mu_{a}=\sum_{a} a \sum_{b} P_{a b} \\
\mu_{b}=\sum_{b} b \sum_{a} P_{a b} \\
\sigma_{a}^{2}=\sum_{a}\left(a-\mu_{a}\right)^{2} \sum_{b} P_{a b} \\
\sigma_{b}^{2}=\sum_{b}\left(b-\mu_{b}\right)^{2} \sum_{a} P_{a b}\end{array}$ \\
\hline
\end{tabular}

where,

$a, b=$ Pixel coordinates on the matrix

level $=$ The range of grayscale value between 0-255 (level $=256)$

$P_{a, b} \quad=$ value of coordinat pixel $a, b$ on matrix GLCM

\section{H. Support Vector Machine}

Support Vector machine is one of the supervised machine learning that is commonly used in classification and regression analysis. SVM was widely introduced by Vapnik in the late 90s [35]. SVM can separate data by forming a hyperplane, maximizing margins and dividing data into different classes. The kernel function in SVM can separate data by looking for a hyperplane as a separator between data and finding the best hyperplane to put data in each class [36].

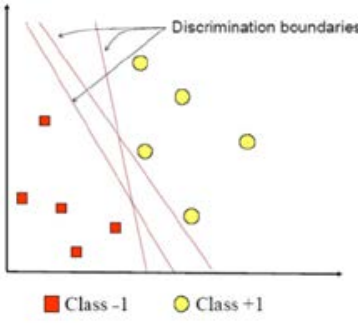

(a)

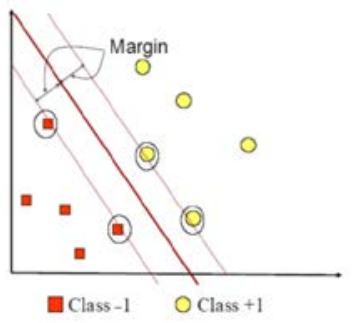

(b)
Fig. 10. Illustration of Class -1 and Class -2 Separated by Hyperplane; a) Several Alternative Lines of Discrimination; b) Hyperplane with the Best Margin.

Fig. 10 shows two different classes between class -1 in red and class +1 in yellow. Fig. 5(a) shows classes -1 and +1 separated by several hyperplanes as differentiator classes. The difference can be obtained by measuring the hyperplane margin and finding the maximum point. Margin is the distance between hyperplanes with the closest pattern of each data class. The pattern that has the closest value is also called the support vector. Fig. 5(b) is two classes of data separated by the best hyperplane, where the hyperplane line is located in the middle between the support vector pattern owned by class -1 and class +1 .

SVM as a classification method has two essential aspects: the first aspect is finding a hyperplane that can optimally separate two classes. The second aspect is the transformation of linearly inseparable classifications into separable ones [35].

Vector learning input and class $\left(x_{-} i, y_{-} i\right)$, where $i=$ $1,2, \ldots$ with $x_{i} \in R^{n}$ and $y \in\{1,-1\}$. In solving problems using linear separation, hyperplane can define boundaries between classes -1 and +1 with the calculation representation as follows. [37]:

$\min _{\omega, b, \xi} \frac{1}{2} \omega^{T} \omega+C \sum_{i=1}^{l} \xi_{i}$

towards subject,

$y_{i}\left(\omega^{T} \phi\left(x_{i}\right)+b\right) \geq 1-\xi_{i} ; \xi_{i} \geq 0$

Solving for classes that cannot be solved linearly, then vector $x_{-} i$ mapped into a higher dimension using the function $\phi$ so that they can be separated linearly. Furthermore, SVM can define a hyperplane that separates linearly with a maximum margin of higher dimensional space. The SVM error parameter is known as $C>0 . K\left(x_{i}, x_{j}\right)=\phi\left(x_{i}\right)^{T}\left(x_{j}\right)$ known as the Kernel function, shown in Table II [38].

TABLE II. SVM KERNEL EQUATION

\begin{tabular}{|l|l|}
\hline Kernel Name & Equation \\
\hline Linear & $K\left(x_{i}, x_{j}\right)=x_{i}{ }^{T} x_{i}$ \\
\hline Polynomial & $K\left(x_{i}, x_{j}\right)=\left(\gamma x_{i}^{T}+x_{j}+r\right)^{d}, \gamma>0$ \\
\hline Gaussian & $K\left(x_{i}, x_{j}\right)=\exp \left(-\frac{\left\|x_{i}-x_{j}\right\|^{2}}{2 \gamma^{2}}\right) \gamma>0$ \\
\hline
\end{tabular}




\section{Results AND ANALYSIS}

In this study, system training was carried out using 75 normal iris data and 75 abnormal iris data. Normal iris data is the iris of people who have no history of heart disease; on the contrary for abnormal iris data is the iris of people who have heart disease.

Fig. 11 shows the training data using linear, polynomial, and gaussian kernel variations. Iris data in training can be separated according to normal (red) and abnormal (blue) classes. The results of linear kernel training separate the data into each class with an even distribution of data. The difference in the polynomial kernel training where the data has been separated and more centralized. The results of the training using the Gaussian Kernel resulted in a tighter grouping than using the two previous kernels. The results of the training are able to separate between classes according to existing characteristics, which can help in the classification of test data and affect the level of recognition accuracy. The further apart the hyperplane in the SVM that separates the classes, the higher the accuracy.

Tests were carried out on 50 normal iris data and 50 abnormal iris data. The data that has gone through the region of interest process is the iris data which reflects the heart. Then this section will be extracted using the GLCM method. The characteristics used in GLCM are Contrast, Dissimilarity,
Homogeneity, Energy, and Correlation. The use of angle variations in the test is carried out to obtain optimal results. For example, Fig. 12 results from GLCM feature extraction at an angle with a distance of 1 .

Taking five types of texture characteristics on iris data is needed to obtain more detailed information in training and testing. Normal and abnormal iris data have different characteristic values. The data can be classified into the appropriate class classification using SVM with three kernels: Linear, Polynomial, and Gaussian.

Simulation testing for variations angle in GLCM $0^{\circ}, 45^{\circ}, 90^{\circ}$ and $135^{\circ}$ with the SVM kernel variation is shown in Fig. 13. By using angle $0^{\circ}$ the highest level of accuracy is obtained by using the Gaussian kernel which reaches 94\%, linear kernel reaches $90 \%$ and Polynomial kernel 88\%. The use of $45^{\circ}$ obtained the highest accuracy rate with $88 \%$ Gaussian kernel, 86\% polynomial kernel and 82\% linear kernel. Then, for angel $90^{\circ}$ the highest level of accuracy is achieved by Gaussian and Linear kernels which reach $92 \%$ and polynomial kernels which reach $88 \%$. Meanwhile, with angle $135^{\circ}$ the highest level of accuracy is achieved by $92 \%$ polynomial kernel, 90\% Gaussian kernel and linear kernel which reaches $84 \%$. From these data, it can be seen that the angle $0^{\circ}$ and $90^{\circ}$ has an average classification accuracy level of $90.67 \%$ at a distance of 1 .

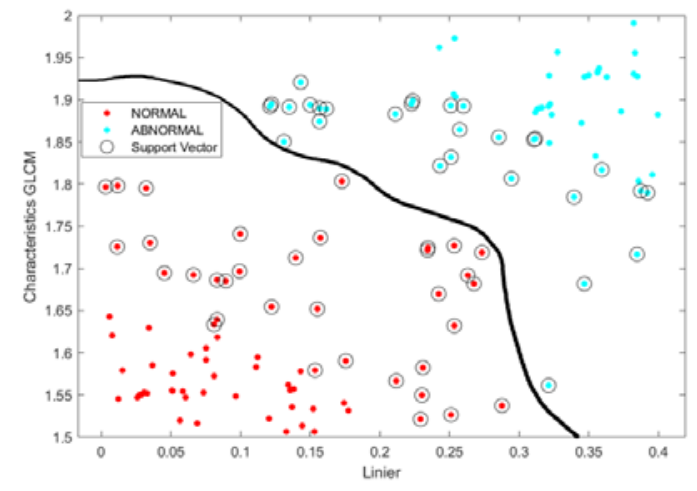

(a)

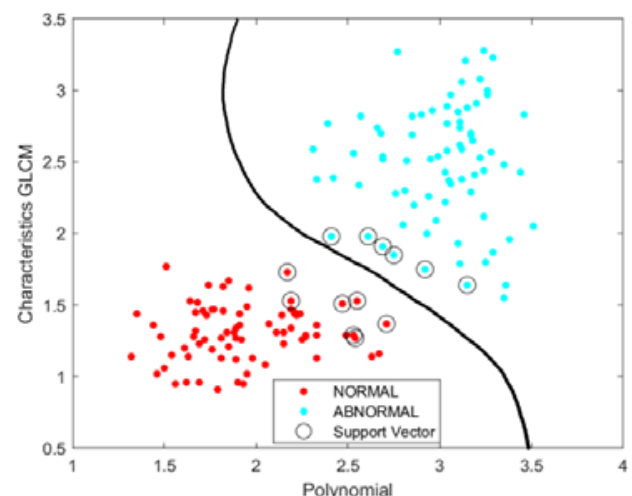

(b)

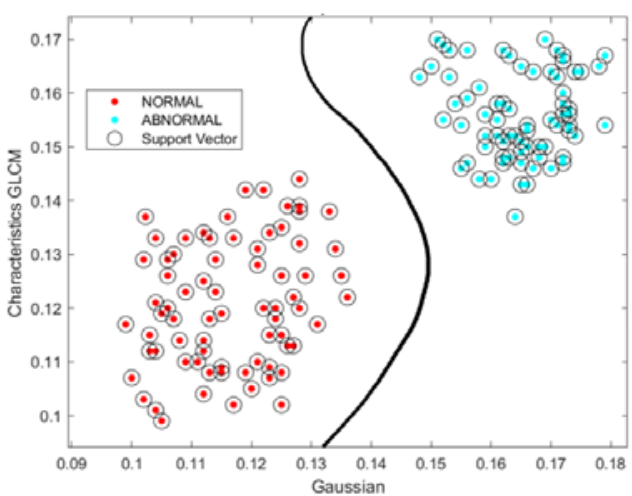

(c)

Fig. 11. Results of Training using Kernel Variations; a) Linear kernel; b) Polynomial kernel; c) Gaussian kernel. 


\begin{tabular}{|c|c|c|c|c|c|c|}
\hline $\begin{array}{c}\text { Iris } \\
\text { Name }\end{array}$ & Contrast & Dissimilarity & Homogeneity & Energy & Correlation & $\begin{array}{l}\text { Output } \\
\text { Target }\end{array}$ \\
\hline iris_1_N & 0.113840 & 1.534803 & 0.875909 & 0.223541 & 0.530184 & Normal \\
\hline iris $2 \mathrm{~N}$ & 0.137012 & 1.556464 & 0.909280 & 0.242990 & 0.567106 & Normal \\
\hline iris_3_N & 0.092625 & 1.514334 & 0.861357 & 0.205152 & 0.509539 & Normal \\
\hline iris_4_N & 0.126065 & 1.547119 & 0.890755 & 0.230201 & 0.536350 & Normal \\
\hline iris_5_N & 0.105937 & 1.530152 & 0.869887 & 0.215905 & 0.521832 & Normal \\
\hline iris_6_N & 0.131387 & 1.554229 & 0.904014 & 0.237140 & 0.561935 & Normal \\
\hline iris $7 \mathrm{~N}$ & 0.108102 & 1.533261 & 0.871327 & 0.217578 & 0.522959 & Normal \\
\hline iris_ 8 - N & 0.108949 & 1.533432 & 0.871362 & 0.218346 & 0.523537 & Normal \\
\hline iris $9 \mathrm{~N}$ & 0.120500 & 1.542982 & $0.8 \$ 4436$ & 0.227554 & 0.532651 & Normal \\
\hline iris_10_N & 0.114233 & 1.536698 & 0.876382 & 0.224434 & 0.530588 & Normal \\
\hline iris_11_N & 0.122934 & 1.545506 & 0.888404 & 0.229146 & 0.533992 & Normal \\
\hline iris_12_N & 0.117435 & 1.540503 & 0.882702 & 0.225038 & 0.531913 & Normal \\
\hline iris_13_N & 0.099871 & 1.525741 & 0.866672 & 0.214070 & 0.518462 & Normal \\
\hline iris $14-\mathrm{N}$ & 0.095196 & 1.518999 & 0.862712 & 0.206916 & 0.513388 & Normal \\
\hline iris_15_N & 0.130812 & 1.553009 & 0.901850 & 0.236055 & 0.557268 & Normal \\
\hline iris_16_N & 0.091719 & 1.511600 & 0.801879 & 0.200253 & 0.505685 & Normal \\
\hline iris_17_N & 0.129111 & 1.550672 & 0.900261 & 0.232182 & 0.544913 & Normal \\
\hline iris_18_N & 0.135080 & 1.555202 & 0.908694 & 0.240291 & 0.565829 & Normal \\
\hline iris $19 \mathrm{~N}$ & 0.099355 & 1.524967 & 0.865813 & 0.208856 & 0.516272 & Normal \\
\hline iris_20_N & 0.122329 & 1.544525 & 0.888029 & 0.228287 & 0.533974 & Normal \\
\hline iris_21_N & 0.128799 & 1.550137 & 0.893426 & 0.231181 & 0.543751 & Normal \\
\hline iris_22_N & 0.106555 & 1.532400 & 0.870643 & 0.216479 & 0.522577 & Normal \\
\hline iris_23_N & 0.121230 & 1.544125 & 0.885671 & 0.227872 & 0.532727 & Normal \\
\hline iris_24_N & 0.113138 & 1.534629 & 0.874729 & 0.222447 & 0.530116 & Normal \\
\hline iris_25_N & 0.117625 & 1.541798 & 0.882963 & 0.225234 & 0.532239 & Normal \\
\hline iris_26_N & 0.102282 & 1.527922 & 0.868529 & 0.214469 & 0.519255 & Normal \\
\hline iris_27_N & 0.099305 & 1.521108 & 0.865209 & 0.207950 & 0.514743 & Normal \\
\hline iris_28_N & 0.112204 & 1.533513 & 0.872597 & 0.219700 & 0.524008 & Normal \\
\hline iris_29_N & 0.138300 & 1.556943 & 0.909989 & 0.244259 & 0.567959 & Normal \\
\hline iris_ $30-\mathrm{N}$ & 0.114878 & 1.537424 & 0.877369 & 0.224701 & 0.530918 & Normal \\
\hline iris_31_N & 0.127546 & 1.550092 & 0.893227 & 0.230965 & 0.542584 & Normal \\
\hline iris_ $32 \_\mathrm{N}$ & 0.093969 & 1.515478 & 0.862557 & 0.205657 & 0.512927 & Normal \\
\hline iris_33_N & 0.132381 & 1.554773 & 0.905114 & 0.238878 & 0.563652 & Normal \\
\hline iris_34_N & 0.126967 & 1.548483 & 0.891537 & 0.230536 & 0.539478 & Normal \\
\hline iris_35_N & 0.115360 & 1.540278 & 0.877390 & 0.224781 & 0.531348 & Normal \\
\hline iris_36_N & 0.112290 & 1.534626 & 0.872953 & 0.220016 & 0.529340 & Normal \\
\hline iris_37_N & 0.129724 & 1.551908 & 0.900730 & 0.235930 & 0.549226 & Normal \\
\hline iris_38_N & 0.135673 & 1.555577 & 0.908772 & 0.241097 & 0.566311 & Normal \\
\hline iris_ 39 N & 0.119169 & 1.542638 & 0.883913 & 0.227447 & 0.532550 & Normal \\
\hline iris_40_N & 0.092119 & 1.511946 & 0.804009 & 0.202494 & 0.505771 & Normal \\
\hline iris_41_N & 0.118351 & 1.541898 & 0.883264 & 0.227327 & 0.532538 & Normal \\
\hline iris_42_N & 0.125965 & 1.547022 & 0.889048 & 0.229819 & 0.536237 & Normal \\
\hline iris_43_N & 0.135992 & 1.556369 & 0.909058 & 0.242795 & 0.566957 & Normal \\
\hline iris $44 \mathrm{~N}$ & 0.103427 & 1.529705 & 0.869788 & 0.214554 & 0.521212 & Normal \\
\hline iris_ $45 \_\mathrm{N}$ & 0.131376 & 1.554161 & 0.903425 & 0.237118 & 0.561623 & Normal \\
\hline iris_46_N & 0.096582 & 1.519643 & 0.863847 & 0.207312 & 0.514044 & Normal \\
\hline iris_47_N & 0.097895 & 1.520501 & 0.864548 & 0.207614 & 0.514577 & Normal \\
\hline iris_48_N & 0.094387 & 1.518236 & 0.862694 & 0.205886 & 0.513336 & Normal \\
\hline iris_49_N & 0.131368 & 1.553203 & 0.903417 & 0.237117 & 0.557788 & Normal \\
\hline iris $50 \mathrm{~N}$ & 0.099767 & 1.525568 & 0.865828 & 0.210194 & 0.517831 & Normal \\
\hline
\end{tabular}

\begin{tabular}{|c|c|c|c|c|c|c|}
\hline Iris Name & Contrast & Dissimilarity & Homogeneity & Energy & Correlation & $\begin{array}{l}\text { Output } \\
\text { Target }\end{array}$ \\
\hline iris_1_AB & 0.329457 & 1.906424 & 0.586281 & 0.437836 & 0.834653 & Abnormal \\
\hline iris_2_AB & 0.357422 & 1.936454 & 0.612558 & 0.457730 & 0.860977 & Abnormal \\
\hline iris_3_AB & 0.311232 & 1.883080 & 0.571065 & 0.415062 & 0.810081 & Abnormal \\
\hline iris_4_AB & 0.343302 & 1.921688 & 0.603311 & 0.447064 & 0.849877 & Abnormal \\
\hline iris_5_AB & 0.322849 & 1.895183 & 0.581332 & 0.433354 & 0.823621 & Abnormal \\
\hline iris_6_AB & 0.351030 & 1.930470 & 0.607544 & 0.450543 & 0.858039 & Abnormal \\
\hline iris_7_AB & 0.323676 & 1.898782 & 0.582187 & 0.434065 & 0.825033 & Abnormal \\
\hline iris_8_AB & 0.325459 & 1.903395 & 0.583222 & 0.434778 & 0.827332 & Abnormal \\
\hline iris_9_AB & 0.337747 & 1.917268 & 0.599114 & 0.441101 & 0.844149 & Abnormal \\
\hline iris_10_AB & 0.331364 & 1.906475 & 0.586905 & 0.438649 & 0.835570 & Abnormal \\
\hline iris_11_AB & 0.342754 & 1.919736 & 0.602187 & 0.444860 & 0.848469 & Abnormal \\
\hline iris_12_AB & 0.333855 & 1.915622 & 0.587599 & 0.438992 & 0.839845 & Abnormal \\
\hline iris_13_AB & 0.321665 & 1.892886 & 0.578839 & 0.430163 & 0.820626 & Abnormal \\
\hline iris_14_AB & 0.312733 & 1.886257 & 0.574593 & 0.422003 & 0.814229 & Abnormal \\
\hline iris_15_AB & 0.348505 & 1.927886 & 0.607177 & 0.449208 & 0.856424 & Abnormal \\
\hline iris_16_AB & 0.309968 & 1.882335 & 0.505143 & 0.413547 & 0.807357 & Abnormal \\
\hline iris_17_AB & 0.346304 & 1.926923 & 0.604156 & 0.448388 & .856050 & Abnormal \\
\hline iris_18_AB & 0.354216 & 1.932049 & 0.609695 & 0.454194 & 0.858625 & Abnormal \\
\hline iris_19_AB & 0.321182 & 1.891907 & 0.578466 & 0.429390 & 0.818582 & Abnormal \\
\hline iris_20_AB & 0.340548 & 1.918924 & 0.600069 & 0.444651 & 0.847406 & Abnormal \\
\hline iris_21_AB & 0.346011 & 1.924694 & 0.604131 & 0.447560 & 0.854405 & Abnormal \\
\hline iris_22_AB & 0.323357 & 1.896666 & 0.581541 & 0.434037 & .824947 & Abnormal \\
\hline iris_23_AB & 0.339513 & 1.918457 & 0.599624 & 0.442365 & 0.844884 & Abnormal \\
\hline iris_24_AB & 0.328327 & 1.905546 & 0.585306 & 0.436282 & 0.834512 & Abnormal \\
\hline iris_25_AB & 0.334689 & 1.916217 & 0.588875 & 0.439787 & 0.840532 & Abnormal \\
\hline iris_26_AB & 0.321787 & 1.894896 & 0.578999 & 0.431165 & 0.823337 & Abnormal \\
\hline iris_27_AB & 0.316530 & 1.890742 & 0.577807 & 0.427436 & 0.817862 & Abnormal \\
\hline iris_28_AB & 0.325815 & 1.903526 & 0.583535 & 0.436119 & 0.829093 & Abnormal \\
\hline iris_29_AB & 0.357517 & 1.937150 & 0.614173 & 0.458252 & 0.861316 & Abnormal \\
\hline iris_30_AB & 0.332150 & 1.911090 & 0.587349 & 0.438668 & 0.838154 & Abnormal \\
\hline iris_31_AB & 0.344889 & 1.924101 & 0.603769 & 0.447470 & 0.853389 & Abnormal \\
\hline iris_32_AB & 0.311703 & 1.883815 & $0.57199 \mathrm{~s}$ & 0.419483 & 0.813329 & Abnormal \\
\hline iris_33_AB & 0.351180 & 1.931843 & 0.608249 & 0.452731 & 0.858070 & Abnormal \\
\hline iris_34_AB & 0.343480 & 1.922127 & 0.603341 & 0.447468 & 0.850663 & Abnormal \\
\hline iris_35_AB & 0.333213 & 1.913168 & 0.587382 & 0.438902 & 0.838449 & Abnormal \\
\hline iris_36_AB & 0.327655 & 1.903563 & 0.585244 & 0.436248 & 0.833949 & Abnormal \\
\hline iris_37_AB & 0.346971 & 1.927239 & 0.605717 & 0.448549 & 0.856358 & Abnormal \\
\hline iris_38_AB & 0.355019 & 1.933204 & 0.609905 & 0.454372 & 0.859139 & Abnormal \\
\hline iris_39_AB & 0.335945 & 1.916977 & 0.593943 & 0.440565 & 0.843881 & Abnormal \\
\hline iris_40_AB & 0.310655 & 1.882698 & 0.568374 & 0.414822 & 0.807485 & Abnormal \\
\hline iris_41_AB & 0.334799 & 1.916866 & 0.589686 & 0.439879 & 0.842201 & Abnormal \\
\hline iris_42_AB & 0.343254 & 1.920767 & 0.602435 & 0.447036 & 0.849754 & Abnormal \\
\hline iris_43_AB & 0.356754 & 1.934325 & 0.611557 & 0.456995 & 0.860948 & Abnormal \\
\hline iris_44_AB & 0.322293 & & 0.579395 & & 0.823568 & \\
\hline iris_45_AB & 0.350102 & 1.929388 & 0.607501 & 0.450050 & 0.857980 & Abnormal \\
\hline iris_46_AB & 0.313168 & 1.889213 & 0.575137 & 0.424492 & 0.815853 & Abnormal \\
\hline iris_47_AB & 0.314682 & 1.890048 & 0.576256 & 0.426567 & 0.817348 & Abnormal \\
\hline iris_48_AB & 0.311746 & 1.885040 & 0.573339 & 0.419889 & 0.813650 & Abnormal \\
\hline iris_49_AB & 0.349897 & 1.929132 & 0.607417 & 0.449636 & 0.856578 & Abnormal \\
\hline iris_50_AB & 0.321245 & 1.892147 & 0.578643 & 0.429447 & 0.819186 & Abnormal \\
\hline
\end{tabular}

Fig. 12. GLCM Characteristic Value using Angle $0^{\circ}$ and $d=1$ on Normal and Abnormal Iris Data.

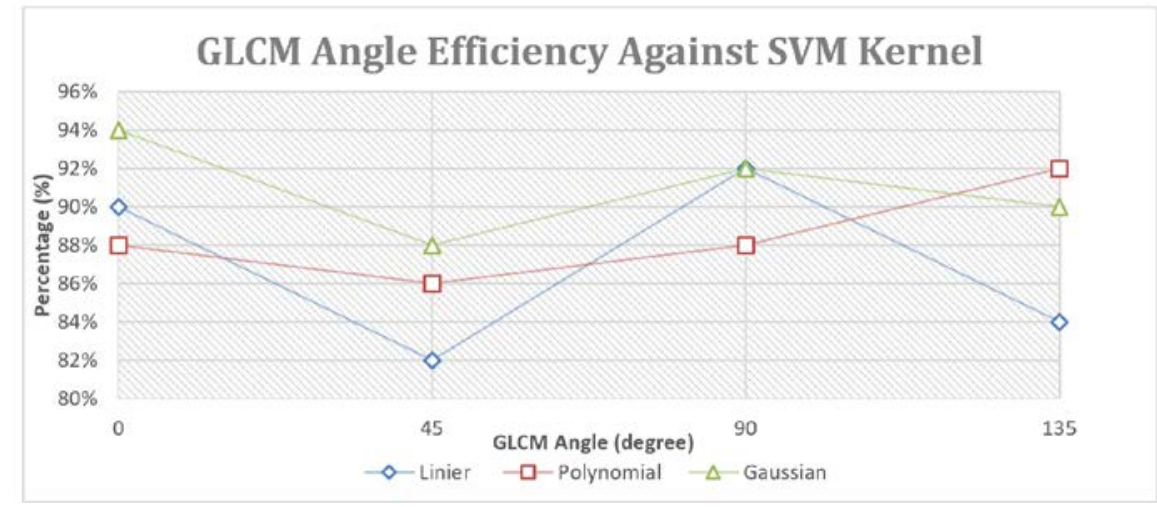

Fig. 13. The Results of the Test with Variations of the GLCM Angle on the Variation of the SVM Kernel.

TABLE III. The AVERAge LeVEl of ANGLE ACCURACY ON THE VARIATION OF THE SVM KERNEL

\begin{tabular}{|l|l|l|}
\hline SVM Classifiers & Training Time (s) & Accuracy Percentage \\
\hline Linier & 381.73 & $87 \%$ \\
\hline Polynomial & 422.28 & $89 \%$ \\
\hline Gaussian & 583.09 & $91 \%$ \\
\hline
\end{tabular}

From Table III, it can be concluded that the average for each angle variation using different SVM kernels has better performance in the classification process. By testing 100 normal and abnormal iris data, a high degree of accuracy was obtained. The use of linear SVM obtained an accuracy rate of $87 \%$, then the use of polynomials obtained $89 \%$. While the use of the kernel with the highest accuracy reaching $91 \%$, was obtained using Gaussian.

Simulation tests with the Gaussian kernel proved to be superior in iris image classification. The Gaussian kernel 
considers the probability of the density function of the standard deviation, squared, and variance. Gaussian can add data space into a higher dimensional vector to determine the intersection of hyperplanes more accurately. With flexible limiting performance, the Gaussian kernel can obtain a higher accuracy level than Linear and Polynomial kernels.

It is proven that normal and abnormal iris conditions can be identified using GLCM feature extraction and a classification process using the Gaussian method on the iris image. Identification is made to assist in providing an early diagnosis of heart conditions through the iris using the iridology method.

\section{CONCLUSION}

This study has proposed a new method to determine the condition of the heart through the iris using the SVM classification with variations of the linear kernel, polynomial kernel, and Gaussian kernel. The use of GLCM characteristics as feature extraction has an essential role in the classification process. The main contribution in this study is not only limited to determining heart health conditions through the iris but also contributes to the automatic processing of the iris with CHT. The proposed system in determining the heart condition automatically is to optimize the classification by using angle $0^{\circ}$ and $90^{\circ}$ on GLCM with SVM classification on the Gaussian kernel to obtain a high level of accuracy. In ongoing research, the iris database can be added to improve the classification to make it more accurate. Different extraction methods can be used to get the results of image extraction with a smaller size so that it can increase the system's speed in iris identification.

\section{ACKNOWLEDGMENT}

This work was supported by the Kariadi Hospital in Semarang and the Department of Engineering at the Universitas Palangkaraya and the Department of Engineering at the Universitas Tanjungpura, Indonesia.

\section{REFERENCES}

[1] Kementrian Kesehatan Republik Indonesia, "Hari Jantung Sedunia (HJS) Tahun 2019: Jantung Sehat, SDM Unggul - Direktorat P2PTM,” P2PTM Kemenkes RI. 2019, [Online]. Available: http://p2ptm.kemkes.go.id/kegiatan-p2ptm/pusat-/hari-jantung-seduniahjs-tahun-2019-jantung-sehat-sdm-unggul.

[2] WHO, "10 Facts on Ageing and Health," Who, vol. 2050, no. May 2017, p. 2014, 2016.

[3] E. E. Tuppo, M. P. Trivedi, J. B. Kostis, J. Daevmer, J. Cabrera, and W. J. Kostis, "The role of public health versus invasive coronary interventions in the decline of coronary heart disease mortality," Ann. Epidemiol., vol. 55, pp. 91-97, 2021, doi: 10.1016/j.annepidem.2020.10.005.

[4] C. Murray, C. Atkinson, K. Bhalla, G. Birbeck, R. Burstein, and D. Chou, "The state of US health, 1990-2010: burden of diseases, injuries, and risk factors.," JAMA - J. Am. Med. Assoc., vol. 310, no. 6, pp. 591608, 2013, doi: 10.1001/jama.2013.13805.The.

[5] Y. H. Chiu et al., "Association between intake of fruits and vegetables by pesticide residue status and coronary heart disease risk," Environ. Int., vol. 132, no. May, p. 105113, 2019, doi: 10.1016/j.envint.2019.105113.

[6] E. G. Nabel and E. Braunwald, "A Tale of Coronary Artery Disease and Myocardial Infarction,” pp. 54-63, 2012.

[7] F. J. Wolters et al., "Coronary heart disease, heart failure, and the risk of dementia: A systematic review and meta-analysis," Alzheimer's
Dement., vol. 14, no. 11, pp. 1493-1504, 2018, doi: 10.1016/j.jalz.2018.01.007.

[8] R. Biswas, J. Uddin, and M. J. Hasan, "A new approach of iris detection and recognition,” Int. J. Electr. Comput. Eng., vol. 7, no. 5, pp. 25302536, 2017, doi: 10.11591/ijece.v7i5.pp2530-2536.

[9] H. Ohmaid, S. Eddarouich, A. Bourouhou, and M. Timouyas, "Iris segmentation using a new unsupervised neural approach,” IAES Int. J. Artif. Intell., vol. 9, no. 1, pp. 58-64, 2020, doi: 10.11591/ijai.v9.i1.pp58-64.

[10] B. Jensen, “Science of Iridology,” pp. 1-2, 1982.

[11] J. Deck, "Principles of Iris Diagnosis," no. June 1985, pp. 1-4.

[12] M. Gopalan and G. S. Pillai, "Human iris patterns - Iridology Applications,” J. Anat. Soc. India, vol. 67, p. S68, Aug. 2018, doi: 10.1016/j.jasi.2018.06.132.

[13] R. B. Esteves, J. A. P. Morero, S. de S. Pereira, K. D. S. Mendes, K. M. Hegadoren, and L. Cardoso, "Parameters to increase the quality of iridology studies: A scoping review,” Eur. J. Integr. Med., vol. 43, p. 101311, Apr. 2021, doi: 10.1016/j.eujim.2021.101311.

[14] P. Samant and R. Agarwal, "Machine learning techniques for medical diagnosis of diabetes using iris images," Comput. Methods Programs Biomed., vol. 157, pp. 121-128, Apr. 2018, doi: 10.1016/j.cmpb.2018.01.004.

[15] R. A. Ramlee and S. Ranjit, "Using iris recognition algorithm, detecting cholesterol presence,” Proc. - 2009 Int. Conf. Inf. Manag. Eng. ICIME 2009, pp. 714-717, 2009, doi: 10.1109/ICIME.2009.61.

[16] K. Gopalakrishna and S. A. Hariprasad, "Real-time fatigue analysis of driver through iris recognition,” Int. J. Electr. Comput. Eng., vol. 7, no. 6, pp. 3306-3312, 2017, doi: 10.11591/ijece.v7i6.pp3306-3312.

[17] L. I. Permatasari, A. Novianty, and T. W. Purboyo, "Heart disorder detection based on computerized iridology using support vector machine,” ICCEREC 2016 - Int. Conf. Control. Electron. Renew. Energy, Commun. 2016, Conf. Proc., pp. 157-161, 2017, doi: 10.1109/ICCEREC.2016.7814983.

[18] R. Aminah and A. H. Saputro, "Application of machine learning techniques for diagnosis of diabetes based on iridology," 2019 Int. Conf. Adv. Comput. Sci. Inf. Syst. ICACSIS 2019, pp. 133-138, 2019, doi: 10.1109/ICACSIS47736.2019.8979755.

[19] I. A. Qasmieh, H. Alquran, and A. M. Alqudah, "Occluded iris classification and segmentation using self-customized artificial intelligence models and iterative randomized Hough transform,” Int. J. Electr. Comput. Eng., vol. 11, no. 5, pp. 4037-4049, 2021, doi: 10.11591/ijece.v11i5.pp4037-4049.

[20] T. Chekouo, S. Mohammed, and A. Rao, “A Bayesian 2D functional linear model for gray-level co-occurrence matrices in texture analysis of lower grade gliomas,” NeuroImage Clin., vol. 28, p. 102437, Jan. 2020, doi: 10.1016/j.nicl.2020.102437.

[21] C. J. C. Burges, “A Tutorial on Support Vector Machines for Pattern Recognition,” Data Min. Knowl. Discov., vol. 2, pp. 121-167, 1998.

[22] D. C. Adelina, R. Sigit, T. Harsono, and M. Rochmad, "Identification Of Diabetes In Pancreatic Organs Using Iridology,” pp. 114-119, 2017.

[23] L. F. Salles and M. J. P. de Silva, "Iridology: A systematic review," Rev. da Esc. Enferm., vol. 42, no. 3, pp. 585-589, 2008, doi: 21201.

[24] S. E. Hussein, O. A. Hassan, and M. H. Granat, "Assessment of the potential iridology for diagnosing kidney disease using wavelet analysis and neural networks," Biomed. Signal Process. Control, vol. 8, no. 6, pp. 534-541, 2013, doi: 10.1016/j.bspc.2013.04.006.

[25] L. S. A. Putra, L. Sumarno, and V. A. Gunawan, "The recognition of semaphore letter code using haar wavelet and euclidean function," Int. Conf. Electr. Eng. Comput. Sci. Informatics, vol. 2018-Octob, no. 1, pp. 759-763, 2018, doi: 10.1109/EECSI.2018.8752707.

[26] A. O. Djekoune, K. Messaoudi, and K. Amara, "Incremental circle hough transform: An improved method for circle detection," Optik (Stuttg)., vol. 133, pp. 17-31, Mar. 2017, doi: 10.1016/j.ijleo.2016.12.064.

[27] T. Lefevre, B. Dorizzi, S. Garcia-Salicetti, N. Lemperiere, and S. Belardi, "Effective elliptic fitting for iris normalization," Comput. Vis. Image Underst., vol. 117, no. 6, pp. 732-745, Jun. 2013, doi: 10.1016/j.cviu.2013.01.005. 
[28] J. Daugman, "High Conf Visual Recog of Persons by a test of statistical significance PAMI93,” Ieee Pami, vol. 15, no. 11, 1993.

[29] G. Indrawan, S. Akbar, and B. Sitohang, "Fingerprint direct-access strategy using local-star-structure-based discriminator features: A comparison study,” Int. J. Electr. Comput. Eng., vol. 4, no. 5, pp. 817830, 2014, doi: 10.11591/ijece.v4i5.6589.

[30] L. S. Ade Putra, R. Rizal Isnanto, A. Triwiyatno, and V. A. Gunawan, "Identification of Heart Disease with Iridology Using Backpropagation Neural Network,” 2018 2nd Borneo Int. Conf. Appl. Math. Eng. BICAME 2018, pp. 138-142, 2018, doi: 10.1109/BICAME45512.2018.1570509882.

[31] R. P. Wildes, "Iris recognition: An emerging biometrie technology," Proc. IEEE, vol. 85, no. 9, pp. 1348-1363, 1997, doi: 10.1109/5.628669.

[32] R. A. Manju, G. Koshy, and P. Simon, "Improved Method for Enhancing Dark Images based on CLAHE and Morphological Reconstruction,” Procedia Comput. Sci., vol. 165, no. 2019, pp. 391398, 2019, doi: 10.1016/j.procs.2020.01.033.

[33] V. Naghashi, "Co-occurrence of adjacent sparse local ternary patterns: A feature descriptor for texture and face image retrieval," Optik (Stuttg)., vol. 157, pp. 877-889, Mar. 2018, doi: 10.1016/j.ijleo.2017.11.160.
[34] D. W. Yang and H. Wu, "Three-dimensional temperature uniformity assessment based on gray level co-occurrence matrix," Appl. Therm. Eng., vol. 108, pp. 689-696, Sep. 2016, doi: 10.1016/j.applthermaleng.2016.07.145.

[35] C. Aroef, Y. Rivan, and Z. Rustam, "Comparing random forest and support vector machines for breast cancer classification,” Telkomnika (Telecommunication Comput. Electron. Control., vol. 18, no. 2, pp. 815-821, 2020, doi: 10.12928/TELKOMNIKA.V18I2.14785.

[36] S. Widodo, R. N. Rohmah, B. Handaga, and L. D. D. Arini, "Lung diseases detection caused by smoking using support vector machine," Telkomnika (Telecommunication Comput. Electron. Control., vol. 17, no. 3, pp. 1256-1266, 2019, doi: 10.12928/TELKOMNIKA.V17I3.9799.

[37] H. Ohmaid, S. Eddarouich, A. Bourouhou, and M. Timouyas, "Comparison between svm and knn classifiers for iris recognition using a new unsupervised neural approach in segmentation,” IAES Int. J. Artif. Intell., vol. 9, no. 3, pp. 429-438, 2020, doi: 10.11591/ijai.v9.i3.pp429-438.

[38] A. Czajka, K. W. Bowyer, M. Krumdick, and R. G. Vidalmata, "Recognition of Image-Orientation-Based Iris Spoofing," IEEE Trans. Inf. Forensics Secur., vol. 12, no. 9, pp. 2184-2196, 2017, doi: 10.1109/TIFS.2017.2701332. 